\title{
Comparing Attention and Cognitive Function in School Children across Noise Conditions: A Quasi-Experimental Study
}

\author{
Soo-young Bhang', Jaekook Yoon², Joohyun Sung², Cheolin Yoo², \\ Changsun $\mathrm{Sim}^{2}$, Changmyung Lee ${ }^{3}$, Jaewon $\mathrm{Lee}^{4}$, and Jiho $\mathrm{Lee}^{2 凶}$ \\ ${ }^{1}$ Department of Psychiatry, Eulji University Eulji General Hospital, Seoul, Republic of Korea \\ ${ }^{2}$ Department of Occupational \& Environmental Medicine, College of Medicine, University of Ulsan, Ulsan University Hospital, Ulsan, Republic of Korea \\ ${ }^{3}$ Laboratory of Noise and Vibration, Department of Mechanics and Automotive Engineering, University of Ulsan, Ulsan, Republic of Korea \\ ${ }^{4}$ Division of Environment \& Noise Research, National Institute of Environmental Research, Seoul, Republic of Korea
}

Objective The effect of acute noise on cognitive function has long been a topic of study, yet these effects remain a serious problem for learning performance in school children.

Methods From November 15, 2010 to December 8, 2010, we enrolled 268 students from three elementary schools (135 boys and 133 girls, 10-12 years old) in Ulsan, Korea. The study subjects were divided into two groups according to their test conditions (background versus additional noise), and tests were conducted using psychological examination tools. Chi-square tests and general linear models were used to assess the differences of impacts on cognition between the two groups.

Results After adjusting for socio-demographic covariates, the noise significantly affected the results of full-scale IQ, verbal IQ, Continuous Performance Test scores, and Children's Color Trails Test and Stroop test scores. The groups at high risk of learning difficulties were more affected by noise than low-risk groups.

Conclusion These findings suggest that noise is hazardous to the attention and performance of elementary school students, particularly for groups at greater risk for poor academic achievement. Additional studies are needed to identify subject-specific levels of noise that can affect attention and cognitive function.

Psychiatry Investig 2018;15(6):620-627

Key Words Noise, Children, Cognition, Attention, Learning, Vulnerability.

\section{INTRODUCTION}

In 2011, the WHO reported the negative impacts of environmental noise on health outcomes as burdens of disease, focusing mainly on cardiovascular disease, cognitive impairment, sleep disturbances, tinnitus, and annoyance. The report mentioned that every year in the EU, cities at least 45,000 disability-adjusted life years are lost for children aged 7-19 years due to environmental noise. These findings compare with at least 22,000 years for tinnitus and 61,000 years

Received: April 27, 2017 Revised: November 15, 2017

Accepted: January 15, 2018

$\triangle$ Correspondence: Jiho Lee, MD, PhD

Department of Occupational \& Environmental Medicine, College of Medicine, University of Ulsan, Ulsan University Hospital, 877 Bangeojinsunhwando-ro, Dong-gu, Ulsan 44055, Republic of Korea

Tel: +82-52-250-7288, Fax: +82-52-250-7289, E-mail: oemdoc@naver.com

(ac) This is an Open Access article distributed under the terms of the Creative Commons Attribution Non-Commercial License (http://creativecommons.org/licenses/bync/4.0) which permits unrestricted non-commercial use, distribution, and reproduction in any medium, provided the original work is properly cited. for ischemic heart disease. ${ }^{1}$ In addition, they recommended that future research on noise will need to focus on vulnerable groups; noise exposure may be worse for certain subgroups, including children, older people, and lower socioeconomic groups.

The cognitive impairment from noise negatively influences children's academic ability. A negative relationship between noise exposure and delayed reading acquisition in young children has been reported. ${ }^{2}$ Children who were chronically exposed to aircraft noise at school have poorer reading ability and memory and poorer performance on standardized tests compared than children who are not exposed. ${ }^{3}$ The largest study of noise and children's cognition undertaken to date is the RANCH project (Road Traffic Noise and Aircraft Noise Exposure and Children's Cognition and Health). This project examined the effects of noise (aircraft and road traffic) on cognition for 2,844 elementary school students (9-10 years) around three large international airports. They found linear exposure-effect relationships between aircraft noise exposure 
at school and children's reading comprehension and recognition memory. ${ }^{4-7}$

Experimental studies have shown the negative impacts of acute exposure on speech perception, listening comprehension, and non-auditory tasks, such as serial recall of visually presented lists and reading. ${ }^{7}$ Studies of the impact of acute noise on reading performance have been mixed. ${ }^{8}$ Acute noise does not affect mathematics performance, but noise might adversely impact the mathematics performance of hyperactive children or children with lower cognitive abilities. ${ }^{9}$ Three studies examined reading and memory in children during acute traffic noise exposure; ${ }^{10-12}$ these studies measured attention capacity, which did not mediate the memory deficits created by noise. Among these three studies, negative impacts due to road traffic noise were reported by only two: Hygge ${ }^{11}$ and Hygge et al. ${ }^{12}$

Dockrell and Shield analyzed the effects of babble and babble mixed with traffic sounds on the performance of third graders on psychological tests, including attention. The overall scores for babble noise were lower than those for quiet conditions. However, the attention test error rates were greater for quiet conditions than those for both noise conditions. ${ }^{13}$ Ljung et al. ${ }^{14}$ examined the effects of road traffic noise and irrelevant speech on children's reading speed, reading comprehension, basic mathematics, and mathematical reasoning among 187 pupils ( 89 girls and 98 boys), 12-13 years old, who were tested in their ordinary classrooms and showed that road traffic noise impaired reading speed $(\mathrm{p}<0.01)$ and basic mathematics $(p<0.05)$. Although the impact of acute noise on cognitive function has been studied extensively, there is still no adequate explanation of the circumstances under which noise affects cognitive performance.

Vulnerable groups can easily be distracted by noise. ${ }^{15}$ Children with language or attention disorders perform worse on auditory tasks than normally developing children exposed to noise due to the mediating roles of linguistic competence and selective attention. ${ }^{16}$ Other researchers have focused on noise exposure in vulnerable groups, ${ }^{15,17-21}$ such as neonates, children younger than 10 years old, and children with autism spectrum disorder. Because the neurocognitive functions associated with academic performance in the classroom are influenced by noise, vulnerable groups with lower academic potential could be more significantly influenced by noise, but there have been no reports on this vulnerable group in school-age children.

The purpose of this study was to investigate the effects of experimental traffic noise ( $>15-17 \mathrm{~dB}$ over background) on the attention and cognition of elementary school students and to evaluate whether vulnerable groups are more substantially influenced by noise disruptions.

\section{METHODS}

\section{Enrollment of the elementary schools}

The three elementary schools in Ulsan were chosen for our study based on the environmental noise level, distance from the traffic road, and socioeconomic status (SES) distribution, according to data from the Office of Education. From November 15, 2010 to December 8, 2010, 268 students from three elementary schools (135 boys and 133 girls; $10-12$ years old) were enrolled in this study. To control for confounders, demographic data on the students from each of the surveyed schools were obtained from parents using a questionnaire. The questionnaire queried the children's medical history, recognition of residential noise, and socioeconomic status. The informed consent forms were approved by the Ulsan University Hospital's Institutional Review Board (UUH-IRB-11-109).

\section{Noise surveys}

The noise levels were measured outside and inside the 3 schools. The main source of noise was road traffic noise. School A was situated three to four kilometers from the airport and $150 \mathrm{~m}$ from a four-lane road in the suburbs; school $\mathrm{B}$ was located approximately $50 \mathrm{~m}$ from a four-lane road on the outskirts of town, and school $\mathrm{C}$ was close to central Ulsan City, situated in a residential area approximately $300 \mathrm{~m}$ from a six-lane road. The average noise levels measured in each school and for the enrolled subjects are shown in Table 1.

Each child was randomly placed in one of two test conditions. The subjects performed a series of attention and cognitive tests while experiencing either background noise or noise exposure. The two noise conditions were as follows.

1) The control group (CG) was exposed to background noise (43.5-46.1 dB) alone and 2) the experimental noisy group (NG) was exposed to an additional 15-17 $\mathrm{dB}$ over the background noise $(60.8-62.8 \mathrm{~dB})$. The noise was a combination of road traffic and aircraft noise.

Before the tests, audiometric tests (pure tone audiometry and tympanometry) were performed to evaluate the hearing threshold levels for all of the children.

\section{Cognitive function tests}

A battery of cognitive function tests was conducted. These

Table 1. The noise levels and participants at the surveyed schools

\begin{tabular}{cccc}
\hline \multirow{2}{*}{ School } & \multicolumn{2}{c}{ Noise levels (LAeq/LCeq, dB) } & \multirow{2}{*}{$\begin{array}{c}\text { Subjects } \\
\text { (male/female) }\end{array}$} \\
\cline { 2 - 3 } & Outside & Inside & \\
\hline A & $54.2 / 64.3$ & $46.0 / 58.5$ & $50 / 39$ \\
B & $58.5 / 67.8$ & $44.0 / 61.3$ & $43 / 44$ \\
C & $62.8 / 66.3$ & $47.9 / 57.0$ & $42 / 50$ \\
\hline
\end{tabular}


tests were appropriate for fourth to sixth grade (10-12 years old) elementary school students. The tests were conducted by a trained nurse using a computerized system.

\section{Korean Wechsler Intelligence Scale for Children, the abbreviated form}

Each child completed the abbreviated form of the Korean Wechsler Intelligence Scale for Children (KEDI-WISC), ${ }^{22}$ which consists of vocabulary, arithmetic, picture arrangement, and block design tests. The scores obtained on the abbreviated batteries are highly correlated with the WISC full-scale intelligence quotient (FSIQ) scores. ${ }^{23}$ The sum of the age-adjusted t-scores for arithmetic and vocabulary was used to estimate the verbal IQ (VIQ), and the scores for the block design and picture arrangement were used to estimate the performance IQ (PIQ).

\section{Continuous Performance Test}

Attention and response inhibition were assessed using a standardized visual version of a computerized continuous performance test called the Comprehensive Attention Test (CAT). ${ }^{24}$ The CAT was standardized for Korean children and adolescents, and its reliability and validity as a diagnostic instrument for attention-deficit/hyperactivity disorder (ADHD) have been studied. ${ }^{25}$ The visual selective attention, auditory selective attention, divided attention, and spatial working memory tests from the CAT were administered to the children.

\section{Stroop Color-Word Test}

The Stroop Color-Word Test ${ }^{26}$ was developed for children 5-14 years old and has been widely applied to assess cognitive inhibition or the ability to ignore the interference from irrelevant stimuli, which mainly reflects frontal lobe function. The Korean version of the Stroop Color-Word Test has been standardized. ${ }^{27}$

\section{Children's Color Trails Test}

The Children's Color Trails Test (CCTT) consists of CCTT1 and CCTT-2. CCTT-1 was used to measure perception ability and attention continuity. CCTT- 2 was used for attention allocation and serial attention processing ability. The two tests are representative measures of complex information processing capabilities.

\section{Korean Learning Disability Evaluation Scale, parent-rated}

The Learning Disability Evaluation Scale (LDES) ${ }^{28}$ consists of 88 items describing the observed characteristics of students with learning disabilities. The Korean version was age-standardized among children. ${ }^{29}$ Seven different age-adjusted subscales of listening, thinking, speaking, reading, writing, spell- ing, and mathematical calculations and the learning quotient (LQ) from the KLDES are used to calculate the LQ as a global measure of learning disabilities. We classified a learning disorder risky group (LD) as those with a LQ below 85 in this study.

\section{Data analysis}

Chi-square tests were used to assess between-school differences in the sex, developmental history, paternal education, and monthly income of each student. General linear model analyses were conducted to assess the differences between the two groups in attention and cognitive functions, after adjusting for confounders.

\section{RESULTS}

The demographic results of this study are summarized in Table 2. Generally, the paternal education level and monthly income at school $\mathrm{C}$ were greater than those at schools A and B. There were significant SES differences among the three schools $\left(\chi^{2}=65.0, \mathrm{p}<0.001\right.$ for the paternal educational levels; $\chi^{2}=33.0, p<0.001$ for the monthly incomes). The hearing levels of the enrolled children were within the reference values (0-20 dBHL, average hearing level $0.5,1$, and $2 \mathrm{kHz})$.

The attention and cognitive performance test scores are summarized in Table 3 , as follows. There were significant differences between the CG and NG on the KEDI-WISC, CAT, CCTT, and Stroop tests (children's version).

For the KEDI-WISC, the arithmetic, vocabulary, picture arrangement subtest, and full-scale IQ scores were significantly different for experimental simulated noise, except for the block design component, after adjusting for socio-demographic covariates. The full-scale IQ scores (116.71 for the CG vs. 109.68 for the NG, $\mathrm{p}<0.001$ ), arithmetic subscores (12.11 for the CG vs. 11.30 for the NG, $\mathrm{p}=0.006$ ), vocabulary subscores (13.37 for the CG vs. 11.80 for the NG, $\mathrm{p}=0.002$ ), and picture arrangement scores (11.59 for the CG vs. 9.99 for the NG, $\mathrm{p}=0.001$ ) were significantly decreased in the NG.

General linear models demonstrated significant differences in the CPT scores between the CG and NG: 1) the response time on the auditory selective attention test differed (85.19 for CG vs. 91.51 for NG, $\mathrm{p}=0.007$ ); 2) the omission error and response time on the divided attention test were different (92.98 for the CG vs. 86.01 for the NG, $\mathrm{p}=0.034$, and 98.84 for the CG vs. 104.72 for the NG, $\mathrm{p}=0.047$, respectively); and 3) the forward memory span and forward correct response on the spatial working memory test were different ( 96.80 for the CG vs. 91.42 for the NG, $\mathrm{p}=0.045$, and 95.21 for the CG vs. 89.23 for the NG, $\mathrm{p}=0.020$, respectively).

For the Stroop test, the word score was significantly affected by the experimental noise (50.82 for the CG vs. 48.22 for 
Table 2. Demographic characteristics of the participants

\begin{tabular}{|c|c|c|c|c|c|}
\hline Characteristic & School A, N (\%) & School B, N (\%) & School C, N (\%) & Total, N (\%) & p-value \\
\hline Grade & & & & & $\chi^{2}=1.058, p=0.901$ \\
\hline 4 th & $29(30.9)$ & $30(31.9)$ & $35(37.2)$ & $94(100)$ & \\
\hline 5 th & $26(30.9)$ & $27(33.8)$ & $27(33.8)$ & $80(100)$ & \\
\hline 6 th & $34(36.2)$ & $30(31.9)$ & $30(31.9)$ & $94(100)$ & \\
\hline Sex & & & & & $\chi^{2}=2.052, p=0.358$ \\
\hline Boys & $50(37.0)$ & $43(31.9)$ & $42(31.1)$ & $135(100)$ & \\
\hline Girls & $39(29.3)$ & $44(33.1)$ & $50(37.6)$ & $133(100)$ & \\
\hline Developmental history & & & & & $\chi^{2}=0.875, p=0.775$ \\
\hline Low birth weight & $5(5.6)$ & $4(4.7)$ & $4(4.3)$ & $13(4.9)$ & \\
\hline Premature baby & $5(5.6)$ & $3(3.5)$ & $5(5.4)$ & $13(4.9)$ & \\
\hline Paternal education & & & & & $\chi^{2}=65.046, p<0.01$ \\
\hline$<8 \mathrm{yr}$ & $4(4.7)$ & $1(1.2)$ & $0(0)$ & $5(1.9)$ & \\
\hline $9-12 \mathrm{yr}$ & $60(69.8)$ & $61(71.8)$ & $24(26.1)$ & $145(55.1)$ & \\
\hline $13-14 \mathrm{yr}$ & $9(10.5)$ & $8(9.4)$ & $13(14.1)$ & $30(11.4)$ & \\
\hline $14-16 \mathrm{yr}$ & $12(14.0)$ & $15(17.6)$ & $50(54.3)$ & $77(29.3)$ & \\
\hline$>17 \mathrm{yr}$ & $1(1.2)$ & $0(0)$ & $5(5.4)$ & $6(2.3)$ & \\
\hline Monthly income (\$) & & & & & $\chi^{2}=33.000, p<0.01$ \\
\hline$<2,000$ & $17(19.1)$ & $26(30.6)$ & $8(8.7)$ & $51(19.2)$ & \\
\hline $2,000-3,000$ & $29(32.6)$ & $24(28.2)$ & 17 (18.5) & $70(26.3)$ & \\
\hline $3,000-4,000$ & $27(30.3)$ & $16(18.8)$ & $23(25.0)$ & $66(24.8)$ & \\
\hline $4,000-5,000$ & $9(10.1)$ & $9(10.6)$ & $22(23.9)$ & $40(15.0)$ & \\
\hline$>5,000$ & $7(7.9)$ & $10(11.8)$ & $22(23.9)$ & $39(14.7)$ & \\
\hline
\end{tabular}

the NG, $\mathrm{p}=0.030$ ). However, the differences in the word scores between the CG and NG showed borderline statistical significance after adjusting for the sex, grade, monthly income, and paternal education level of each student (50.29 for the CG vs. 48.03 for the NG, $\mathrm{p}=0.056$ ). For the CCTT tests, the CCTT-1 total time of the NG was significantly delayed compared with the CG (20.51 for the CG vs. 22.50 for the NG, $\mathrm{p}=0.021$ ).

In addition to these analyses, we divided the participants into four groups, according to the Learning Disorder Scale (LD Risky and non-Risky groups) and noise exposure (CG and NG). As shown in Figure 1, there were significant differences between the noise $+\mathrm{LD}$ risky group and the other groups for the attention subtasks, such as the commission error (Visual Attention Test), reaction time standard deviation variability (Visual Attention Test), commission error (Auditory Attention Test), and commission error for the Divided Attention Test (Visual+Auditory). Additionally, there were significant differences between the N+LD risky group and the other groups for spatial working memory (forward memory span, forward correct response, backward memory span, and backward correct response), as shown in Figure 2.

\section{DISCUSSION}

The objective of this study was to investigate the impacts of experimental traffic noise (approximately 15-17 dB higher than background noise) on the attention and cognitive functions of elementary school students. The comparisons of a battery of tests showed that experimental traffic noise negatively impacted the attention and cognitive performances of children after controlling for their socioeconomic status. In addition, the negative impacts were more prominent in the lower academic performance group.

Regarding IQ scores, according to the KEDI-WISC, the full-scale and verbal IQ scores were affected by experimental traffic noise. Few studies have examined the impact of acute environmental noise on IQ scores. However, the students with relatively low verbal and nonverbal intellectual abilities had lower performance in multisensory integration under either the quiet or noise conditions. Previous studies on the impact of noise on IQ scores showed that lower IQ groups were affected by noise for multisensory integration. ${ }^{30}$ In our study, there were lower IQ scores in the environmental traffic noise group after adjusting the socioeconomic variables. This finding aligned with the results of the controlled tests done by 
Table 3. Comparisons of the K-WISC, CPT, STROOP, and CCTT scores between the Control and Noise groups, after adjusting for sex, grade, monthly income, and paternal education

\begin{tabular}{|c|c|c|c|c|}
\hline \multirow{2}{*}{ Test } & \multirow{2}{*}{ Subtest } & Control group & Noise group & \multirow{2}{*}{$\mathrm{p}$-value } \\
\hline & & Mean (SE) & Mean (SE) & \\
\hline \multirow[t]{5}{*}{ K-WISC } & Arithmetic & $12.11(0.18)$ & $11.30(0.22)$ & 0.006 \\
\hline & Vocabulary & $13.37(0.33)$ & $11.80(0.38)$ & 0.002 \\
\hline & Block design & $14.01(0.34)$ & $13.50(0.40)$ & 0.346 \\
\hline & Picture arrangement & $11.59(0.29)$ & $9.99(0.34)$ & 0.001 \\
\hline & Full-scale IQ & $116.54(1.11)$ & $110.04(1.29)$ & $<0.001$ \\
\hline \multirow[t]{20}{*}{$\mathrm{CPT}$} & Visual selective attention test & & & \\
\hline & Commission error & $90.40(2.25)$ & $92.14(2.61)$ & 0.617 \\
\hline & Omission error & $85.63(1.96)$ & $82.94(2.28)$ & 0.375 \\
\hline & Response time (sec) & $92.37(1.25)$ & $90.73(1.46)$ & 0.400 \\
\hline & Response time-SD & $87.23(2.05)$ & $84.46(2.38)$ & 0.381 \\
\hline & Auditory selective attention test & & & \\
\hline & Commission error & $99.51(1.65)$ & $100.35(1.91)$ & 0.743 \\
\hline & Omission error & $94.55(1.43)$ & $93.87(1.65)$ & 0.757 \\
\hline & Response time (sec) & $85.19(1.50)$ & $91.51(1.74)$ & 0.007 \\
\hline & Response time-SD & $92.01(1.57)$ & $93.04(1.82)$ & 0.671 \\
\hline & Divided attention test & & & \\
\hline & Commission error & $71.49(2.55)$ & $67.23(2.95)$ & 0.279 \\
\hline & Omission error & $92.98(2.12)$ & $86.01(2.46)$ & 0.034 \\
\hline & Response time (sec) & $98.84(1.91)$ & $104.72(2.22)$ & 0.047 \\
\hline & Response time-SD & $97.20(1.84)$ & $96.46(2.13)$ & 0.794 \\
\hline & Spatial working memory test & & & \\
\hline & Forward memory span & $96.80(1.73)$ & $91.42(2.01)$ & 0.045 \\
\hline & Forward correct response & $95.21(1.66)$ & $89.23(1.92)$ & 0.020 \\
\hline & Backward memory span & $89.84(2.46)$ & $85.27(2.85)$ & 0.229 \\
\hline & Backward correct response & $89.80(2.09)$ & $85.43(2.43)$ & 0.176 \\
\hline \multirow[t]{4}{*}{ Stroop } & Word score & $50.29(0.76)$ & $48.03(0.88)$ & 0.056 \\
\hline & Color score & $50.35(0.95)$ & $49.93(1.11)$ & 0.779 \\
\hline & Color-word score & $49.20(0.93)$ & $49.06(1.08)$ & 0.921 \\
\hline & Interference score & $48.36(0.98)$ & $48.65(1.15)$ & 0.851 \\
\hline \multirow[t]{3}{*}{ CCTT } & CCTT-1 total time (T score) & $47.73(1.08)$ & $43.94(1.31)$ & 0.030 \\
\hline & CCTT-2 total time (T score) & $48.63(1.02)$ & $47.26(1.24)$ & 0.400 \\
\hline & Interference score (T score) & $52.22(3.01)$ & $56.76(3.64)$ & 0.343 \\
\hline
\end{tabular}

K-WISC: Korean Wechsler Intelligence Scale for Children, CPT: Continuous Performance Test, STROOP: Stroop Color-Word Test, CCTT: Children's Color Trails Test, IQ: Intelligence Quotient

Shield \& Dockrell that demonstrated that classroom babble (at a steady level of $65 \mathrm{~dB}[\mathrm{~A}] \mathrm{LAeq}$ ) has a detrimental effect on children's performance in verbal and nonverbal tasks. In their study, the levels of environmental noise events from various sources (e.g., sirens and trucks) at random intervals (at a level of $58 \mathrm{~dB}[\mathrm{~A}]$ LAmax noise) were similar to those in our study. However, Barutchu et al. ${ }^{31}$ did not find a significant relationship between the cognitive test results in a noisy envi- ronment and nonverbal IQ in children when IQ was measured using Raven's Progressive Matrices Test.

In our CPT analysis of the reaction speed, the reaction times for the auditory attention test (using a simple auditory cue) and the divided attention test (consisting of both visual and auditory cues) were faster in the noise group. Therefore, children exposed to noise can have enhanced performance for their reaction speeds with auditory stimuli; however, in more 


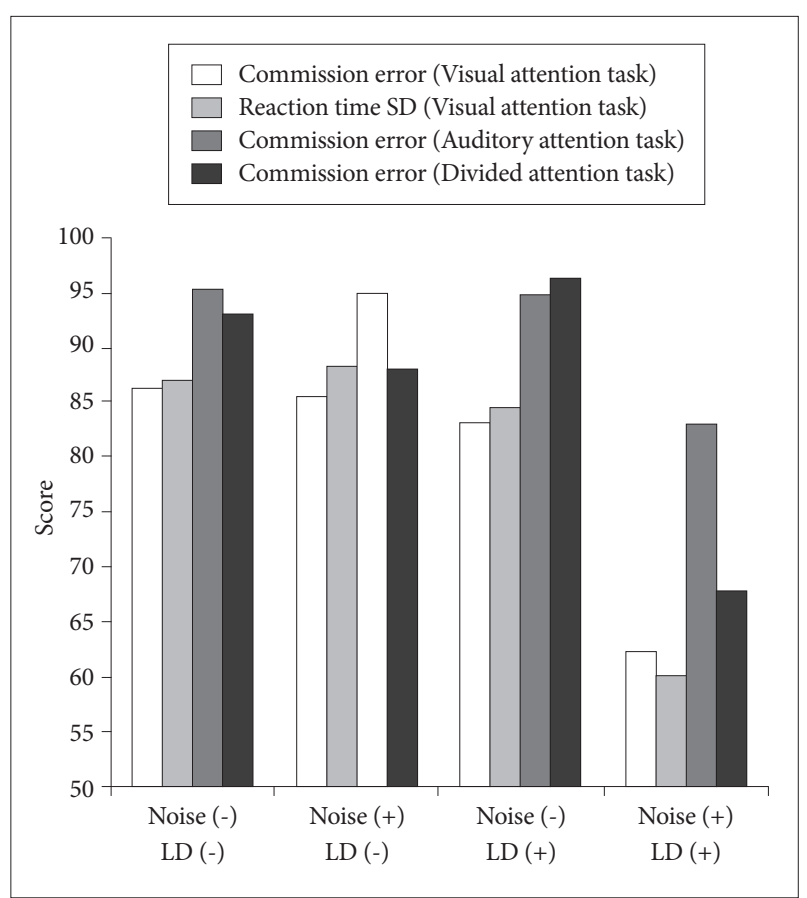

Figure 1. Differences between the noise+LD risky group and the other groups in the attention subtasks, such as the commission error (Visual Attention Test), reaction time standard deviation variability (Visual Attention Test), commission error (Auditory Attention Test), and commission error for the Divided Attention Fest (Visual+ Auditory). LD: learning disability.

complicated tasks with visual cues alone, they are more likely to experience a delay in neurocognitive processing. Barutchu et al. ${ }^{31,32}$ reported that children's motor reaction times were significantly faster for audiovisual stimuli and that the effect is age-related (younger than 12 years old). Barutchu et al. ${ }^{30}$ also demonstrated that auditory background noise has a greater adverse effect on audiovisual integration in children than in adults. Children seem to have more immature multisensory integration and are thus more susceptible to the effects of noisy environments.

One explanation for their faster reaction times in auditory tasks during the noise situation is found in the Moderate Brain Arousal model, ${ }^{33}$ which reported that noise can have some beneficial effect on cognitive performance depending on the neurotransmitter dopamine level. However, they reported that for children (with or without an ADHD diagnosis) who performed a set of short-term memory tasks, there was an antagonistic interaction between noise and the activity performance in the participant group. The noise had a small but beneficial effect on recall for the ADHD-group, whereas the opposite pattern was observed in the controls. However, several investigators found that noise was particularly detrimental to cognitive processing in children with ADHD. However, differences in the reaction time for the visual attention test in our study were insignificant, and the CCTT-1 time was de-

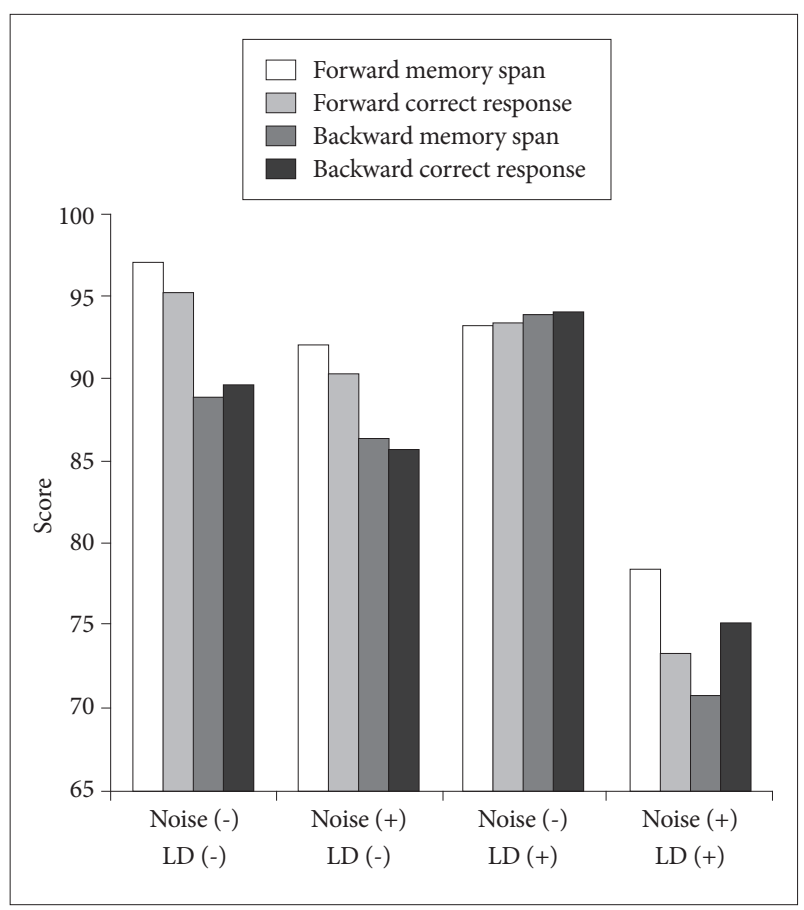

Figure 2. Differences between the N+LD risky group and the other groups for spatial working memory (forward memory span, forward correct response, backward memory span, and backward correct response). N: noise, LD: learning disability.

layed with noise, suggesting that noise can exert a beneficial effect on cognitive functions in response to the auditory stimuli, whereas other cognitive functions will be negatively affected. In addition, the omission errors in the divided attention task were negatively associated with the noise condition, which was in concordance with the fast reaction time in our $\mathrm{CPT}$ results. This result indicates that there is inefficient acceleration of mental processing with errors.

In the spatial working memory component of the CPT test, the forward memory span and the forward correct response were affected by the noisy condition. A possible explanation is that exposure to experimental traffic noise produced observable increases in distractions and off-task times. ${ }^{34}$ In addition, the word score for the Stroop test and the CCTT-1 total time for the noise group were significantly affected by exposure to the experimental traffic noise. These tests are used to assess frontal lobe function, such as attention, cognitive inhibition, and mental operation speed. Both the Stroop and CCTT tests indicated that reading speeds were significantly affected by the experimental traffic noise. These results are in agreement with the results of earlier studies that reported a negative relationship between reading and noise.,12

Some studies have reported that for the groups with attention problems, cognitive performance can be moderated differently compared with that in normal groups by adding external background white noise stimulation. ${ }^{35-37}$ Other studies 
have reported that students with learning disorders experience speech perception deficits in response to noise exposure. In addition, several other researchers have focused on the risky groups that are vulnerable to noise exposure, such as neonates, children younger than 10 years old, and children with autism spectrum disorder. ${ }^{15,17-21}$ In this study, we divided the participants into four groups, according to the Standardized Learning Disability scale and noise level and found that the children who were at risk of learning disabilities were significantly affected by noise conditions compared with the other groups. Therefore, we must think more carefully about the vulnerable groups and make plans to teach them in less noisy conditions, thereby avoiding classroom distractions.

This study has some limitations. We used only two levels of traffic noise (background and experimental noise); therefore, the effects of other noise levels are still unknown. Further study will be needed to assess other environmental noise, such as from aircraft, railway systems, and babble at various noise levels. Furthermore, other neurocognitive functions must be examined using neurobiological measures to assess various memory and emotional changes. Finally, vulnerable populations in particular should have their noise levels reduced in academic environments.

Together, the results demonstrate that noise affects attention and cognitive functions, as measured by the CAT, KEDIWISC, CCTT, and Stroop tests. Moreover, the impacts were consistent after controlling for demographic confounders. These findings suggest that noise (approximately $15-17 \mathrm{~dB}$ higher than background) is hazardous to the attention and cognitive performances of elementary school students, particularly for vulnerable groups with low academic potential. Further study is needed to elucidate the effects of various noise levels on cognitive function.

\section{Acknowledgments}

The research reported here was supported by the National Institute of Environmental Research, Incheon, Korea (2011-0916). The authors are most grateful to Ms. Park Young-ja for all the data collection and technical assistance and to the all of members of the Laboratory of Noise and Vibration, Department of Mechanics and Automotive Engineering, University of Ulsan, for conducting the noise exposure procedures.

\section{REFERENCES}

1. World Health Organization Regional Office for Europe. Burden of Disease from Environmental Noise. Quantification of Healthy Life Years Lost in Europe. Geneva: WHO; 2011.

2. Evans G, Lepore S. Nonauditory effects of noise on children: a critical review. Child Environ 1993;10:31-51.

3. Clark C, Sorqvist P. A 3 year update on the influence of noise on performance and behavior. Noise Health 2012;14:292-296.

4. Clark C, Martin R, van Kempen E, Alfred T, Head J, Davies HW, et al. Exposure-effect relations between aircraft and road traffic noise exposure at school and reading comprehension: the RANCH project. Am J Epidemiol 2006;163:27-37.
5. Stansfeld SA, Berglund B, Clark C, Lopez-Barrio I, Fischer P, Ohrstrom E, et al. Aircraft and road traffic noise and children's cognition and health: a cross-national study. Lancet 2005;365:1942-1949.

6. Matheson M, Clark C, Martin R, van Kempen E, Haines M, Barrio IL, et al. The effects of road traffic and aircraft noise exposure on children's episodic memory: the RANCH project. Noise Health 2010;12:244254.

7. Stansfeld S, Clark C. Health effects of noise exposure in children. Curr Environ Health Rep 2015;2:171-178.

8. Bistrup ML, Hygge S, Keiding L, Passchier-Vermeer W. Health Effects of Noise on Children and Perception of Risk of Noise. Copenhagen: National Institute of Public Health; 2001.

9. Johansson CR. Effects of low intensity, continuous and intermittent noise on mental performance and writing pressure of children with different intelligence and personality characteristics. Ergonomics 1983;26: 275-288.

10. Boman E. The effects of noise and gender on children's episodic and semantic memory. Scand J Psychol 2004;45:407-416.

11. Hygge S. Classroom experiments on the effects of different noise sources and sound levels on long-term recall and recognition in children. Appl Cogn Psychol 2003;17:895-914.

12. Hygge S, Evans GW, Bullinger M. A prospective study of some effects of aircraft noise on cognitive performance in schoolchildren. Psychol Sci 2002;13:469-474.

13. Dockrell JE, Shield BM. Acoustical barriers in classrooms: the impact of noise on performance in the classroom. Brit Educ Res J 2006;32: 509-525.

14. Ljung R, Sorqvist P, Hygge S. Effects of road traffic noise and irrelevant speech on children's reading and mathematical performance. Noise Health 2009;11:194-198.

15. van Kamp I, Davies H. Noise and health in vulnerable groups: a review. Noise Health 2013;15:153-159.

16. Klatte M, Bergström K, Lachmann T. Does noise affect learning? A short review on noise effects on cognitive performance in children. Front Psychol 2013;4:578.

17. Linares C, Diaz J, Tobias A, De Miguel JM, Otero A. Impact of urban air pollutants and noise levels over daily hospital admissions in children in Madrid: a time series analysis. Int Arch Occup Environ Health 2006;79:143-152.

18. Bockelbrink A, Willich SN, Dirzus I, Reich A, Lau S, Wahn U, et al. Environmental noise and asthma in children: sex-specific differences. J Asthma 2008;45:770-773.

19. Byers JF, Waugh WR, Lowman LB. Sound level exposure of high-risk infants in different environmental conditions. Neonatal Netw 2006; 25:25-32.

20. Liu WF; NIC/Q 2005 Physical Environment Exploratory Group. The impact of a noise reduction quality improvement project upon sound levels in the open-unit-design neonatal intensive care unit. J Perinatol 2010;30:489-496.

21. Russo N, Zecker S, Trommer B, Chen J, Kraus N. Effects of background noise on cortical encoding of speech in autism spectrum disorders. J Autism Dev Disord 2009;39:1185-1196.

22. Kwak KJ, Park HW, Kim CT. A study for the standaridization of Korean WISC-III(I). Korean J Dev Psychol 2002;1:19-33.

23. Kaufman AS. A four test short form of the WISC-R. Comtemp Educ Psychol 1976;1:180-196.

24. Yoo HK, Lee J, Kang SH, Park EH, Jung J, Kim BN, et al. Standardization of the comprehensive attention test for the Korean children and adolescents. J Kor Acad Child Adolesc Psychiatry 2009;20:68-75.

25. Suh JM, Lee JS, Kim SY, Kim HW. Standardization of the comprehensive attention test for the Korean children and adolescents. J Kor Acad Child Adolesc Psychiatry 2011;22:246-252.

26. Golden CJ. The Stroop Color and Word Test. Chicago, IL: Stoelting Company; 1978.

27. Shin MS, Park MJ. STROOP: Color and Word Test Children's Version 
for Ages 5-14. Seoul: Hakjisa; 2007.

28. McCarney SB. The Learning Disability Evaluation Scale-renormed. Columbia, MO: Hawthorne Educational Services; 1996.

29. Shin MS, Hong KE, Kim ZS, Cho SC. A standardization study of the Korean version of Learning Disability Evaluation Scale. J Korean Neuropsychiatr Assoc 1998;37:1233-1245.

30. Barutchu A, Crewther SG, Fifer J, Shivdasani MN, Innes-Brown H, Toohey S, et al. The relationship between multisensory integration and IQ in children. Dev Psychol 2011;47:877-885.

31. Barutchu A, Crewther DP, Crewther SG. The race that precedes coactivation: development of multisensory facilitation in children. Dev Sci 2009;12:464-473.

32. Barutchu A, Danaher J, Crewther SG, Innes-Brown H, Shivdasani MN, Paolini AG. Audiovisual integration in noise by children and adults. J
Exp Child Psychol 2010;105:38-50

33. Soderlund G, Sikstrom S, Smart A. Listen to the noise: noise is beneficial for cognitive performance in ADHD. J Child Psychol Psychiatry 2007;48:840-847.

34. Wyon DP. Studies of children under imposed noise and heat stress. Ergonomics 1970;13:598-612.

35. Soderlund GB, Sikstrom S, Loftesnes JM, Sonuga-Barke EJ. The effects of background white noise on memory performance in inattentive school children. Behav Brain Funct 2010;6:55.

36. Ziegler JC, Pech-Georgel C, George F, Lorenzi C. Speech-perceptionin-noise deficits in dyslexia. Dev Sci 2009;12:732-745.

37. Ziegler JC, Pech-Georgel C, George F, Alario FX, Lorenzi C. Deficits in speech perception predict language learning impairment. Proc Natl Acad Sci U S A 2005;102:14110-14115. 